IRRITABLE BOWEL SYNDROME

\title{
T-bet upregulation and subsequent interleukin 12 stimulation are essential for induction of Th1 mediated immunopathology in Crohn's disease
}

\author{
K Matsuoka, N Inoue, T Sato, S Okamoto, T Hisamatsu, Y Kishi, A Sakuraba, O Hitotsumatsu, \\ H Ogata, K Koganei, T Fukushima, T Kanai, M Watanabe, H Ishii, T Hibi
}

Gut 2004;53:1303-1308. doi: 10.1136/gut.2003.024190

See end of article for authors' affiliations

Correspondence to: Dr T Hibi, Department of Internal Medicine, School of Medicine, Keio University, 35

Shinanomachi, Shinjukuku, Tokyo 160-8582,

Japan; rei-ibd@

sc.itc.keio.ac.jp

Revised version received 29 January 2004

Accepted for publication

3 March 2004
Background and aims: Many lines of evidence suggest that T helper cell type 1 (Th1) immune responses predominate in Crohn's disease (CD). Recently, a novel transcription factor T-box expressed in T cells (T-bet) has been reported as the master regulator of Th1 development. This study was designed to investigate the role of T-bet and proinflammatory cytokines in Th1 mediated immunopathology in CD. Materials: CD4+ lamina propria mononuclear cells (LPMCs) were isolated from surgically resected specimens (CD, $n=10$; ulcerative colitis (UC), $n=10$; normal controls $(N L), n=5)$.

Methods: (1) T-bet expression of CD4+ LPMCs was examined by quantitative real time polymerase chain reaction and western blotting. (2) T-bet expression of LPMCs stimulated by interleukin (IL)-12/IL-18 was analysed by western blotting. (3) Interferon $\gamma$ (IFN- $\gamma$ ) production and T-bet expression of CD4+ peripheral blood mononuclear cells (PBMCs) were examined with or without stimulation by anti-CD3/CD28 monoclonal antibodies and/or IL-12.

Results: (1) T-bet expression of CD4+ LPMCs was increased in CD compared with UC and NL. (2) Synergistically, augmentation of IFN- $\gamma$ production by IL-12/IL-18 was independent of T-bet expression in LPMCs. (3) T-bet was induced by T cell receptor stimulation in CD4+ PBMCs. T-bet induction correlated with IFN- $\gamma$ production and with augmentation of surface expressed IL-12 receptor $\beta 2$. Conclusions: T-bet induction by antigenic stimulation and subsequent stimulation by macrophage derived IL-12/IL-18 are important for establishing Th1 mediated immunopathology in CD. rohn's disease $(C D)$ is a chronic inflammatory process involving the gastrointestinal tract, characterised by discontinuous and transmural inflammation. Although the aetiology of CD is not fully understood, accumulating evidence suggests that dysregulation of the local immune system is pivotal in the pathogenesis of CD. ${ }^{12}$ Studies from humans and experimental murine colitis models indicate that in $\mathrm{CD}$, the local immune response tends to be predominantly $\mathrm{T}$ helper cell type 1 (Thl) and is reflected by local release of cytokines such as tumour necrosis factor (TNF)- $\alpha$, interleukin (IL)-12, and IL-18. ${ }^{3-7}$ Studies conducted in experimental murine colitis models showing that neutralising antibodies against TNF- $\alpha$, IL-12, or IL-18 prevent the onset of colitis lend further support to a Thl predominant immunopathology in $\mathrm{CD}^{8-10}$ Indeed, antihuman TNF- $\alpha$ monoclonal antibody (mAb) (Infliximab) is effective for many patients with CD refractory to conventional therapy. ${ }^{11} 12$ Collectively, these observations indicate that Thl mediated immunopathology plays a central role in induction and perpetuation of intestinal inflammation in CD.

Given the observed polarised nature of $\mathrm{T}$ cells in $\mathrm{CD}$, understanding the mechanisms that lead to the establishment of this polarised state in the gastrointestinal mucosa is critical. Naïve CD4 T lymphocytes in transit to becoming either Th1 or Th2 effector cells undergo sequential stages of cytokine activation, commitment, silencing, and physical stabilisation during polarisation into differentiated effector subsets, a process tightly controlled by regulatory transcription factors. ${ }^{13}{ }^{14}$ Various transcription factors such as c-maf, GATA-3, and STAT-6 have been shown to promote expression of several Th2 cytokines, including IL-4, IL-5, and IL-13, either by transactivation of cytokine gene promoters and enhancers or induction of chromatin remodelling. ${ }^{15-17}$ In contrast with Th2 differentiation, very little is known about the molecular basis of Thl differentiation. STAT-4 and IRF- 1 are specifically associated with IL-12 and interferon (IFN) $-\gamma$ signalling in $\mathrm{T}$ cells, respectively, and play a key role in regulating cytokine production of Thl cells at the transcriptional level. ${ }^{13} 18$

Recently, $\mathrm{T}$ box expressed in $\mathrm{T}$ cells (T-bet), a member of the T-box family of transcription factors, has been shown to transactivate the $I F N-\gamma$ gene. ${ }^{19}$ T-bet, whose expression is primarily limited to the immune system, is rapidly induced in early developing Thl cells and is absent in developing Th2 cells. ${ }^{19}$ T-bet deficient mice show normal lymphoid development but exhibit marked impairment in mounting Thl mediated immune responses in response to IL-12. ${ }^{20}$ Moreover, retroviral mediated expression of T-bet in Th2 cells leads to induction of a Thl cytokine profile in these cells. ${ }^{19}$ Thus T-bet initiates Thl cell differentiation by activating Thl genetic programmes and repressing Th2 programmes.

Neurath et al have demonstrated that expression of T-bet is increased in $\mathrm{CD}$ and that $\mathrm{T}$-bet regulates the mucosal cytokine balance in various murine experimental colitis models. ${ }^{21}$ However, the pathophysiological role of T-bet in human CD has not been fully described. In the present study,

Abbreviations: $C D$, Crohn's disease; UC, ulcerative colitis; NL, normal control; IL, interleukin; IL-12R 32 , IL-12 receptor $\beta 2$; LPMC, lamina propria mononuclear cell; PBMC, peripheral blood mononuclear cell; T-bet, T-box expressed in T cells; TCR, T cell receptor; Th1, T helper cell type 1 ; IFN- $\gamma$, interferon $\gamma ; \mathrm{mAb}$, monoclonal antibody; TNF- $\alpha$, tumour necrosis factor $\alpha$; PCR, polymerase chain reaction; AU, arbitrary units; TGF- $\beta$, transforming growth factor $\beta$ 
we investigated the role of T-bet in induction of Thl responses in human $\mathrm{CD}$, and differences in immune responses between $\mathrm{CD}$ and ulcerative colitis (UC).

\section{MATERIALS AND METHODS Patients and samples}

Mucosal samples were obtained from surgically resected inflamed areas of intestinal specimens from 10 patients with $\mathrm{CD}$ and 10 with UC. Patient profiles are summarised in table 1. CD or UC was diagnosed based on clinical, radiographic, endoscopic, and histological findings by established criteria. ${ }^{22}$ The degree of inflammation was histologically moderate to severe in all samples. Normal controls (NL) included mucosal samples from macroscopically and microscopically unaffected areas from patients with sporadic colon cancer. All experiments were approved by the local ethics committees. Informed consent was obtained from all patients before obtaining the samples.

\section{RNA extraction and quantitative real time polymerase chain reaction (PCR)}

Total RNA was extracted using an RNeasy Mini Kit (Qiagen, Hilden, Germany). Total RNA was treated with Qiagen DNase (Qiagen) to remove any contaminating genomic DNA. Complementary DNA (cDNA) was synthesised using the Superscript first strand synthesis system for reverse transcription-PCR (Invitrogen, Carlsbad, California, USA) according to the manufacturer's instructions. Quantitative real time PCR was performed using SYBR Green PCR master mix (Applied Biosystems, Foster City, California, USA) with an ABI PRISM 7700 Sequence Detection System (Applied Biosystems). We confirmed that non-specific bands were not detected by melting curve analysis for each primer set. T-bet mRNA transcripts were normalised with $\beta$-actin mRNA transcripts and expressed as arbitrary units (AU). PCR primers were as follows: T-bet forward, 5'-CCC CCA AGG AAT TGA CAG TTG-3'; reverse 5'-GGG AAA CTA AAG CTC ACA AAC-3'. $\beta$-Actin forward, 5'-AAG CAG GAG TAT GAC GAG TCC G-3'; reverse, 5' -CGG AAC TAA GTC ATA GTC CGC C-3'

\section{Preparation of lamina propria mononuclear cells (LPMCs) and peripheral blood mononuclear cells (PBMCs)}

LPMCs were isolated from surgically resected intestinal specimens using enzymatic techniques, as previously described. ${ }^{3}$ Briefly, dissected mucosa was incubated in calcium and magnesium free Hanks' balanced salt solution (Sigma, St Louis, Missouri, USA) containing 2.5\% fetal bovine serum (BioSource, Camarillo, California, USA) and $1 \mathrm{mM}$ dithiothreitol (Sigma). The mucosa was then

Table 1 Clinical profiles of Crohn's disease (CD) and ulcerative colitis (UC) patients

\begin{tabular}{lll}
\hline & CD & UC \\
\hline No of patients & 10 & 10 \\
Sex (F/M) & $4 / 6$ & $6 / 4$ \\
Age (y) (mean (range)) & $33.0(21-51)$ & $39.4(22-70)$ \\
Disease activity & & \\
CDAl (mean (range)) & $224.8(165-324)$ & - \\
CAl (mean (range)) & - & $9.2(5-16)$ \\
Medication & 9 & 8 \\
5-ASA or SASP & 2 & 3 \\
Steroid & 3 & 1 \\
Azathioprine & \multicolumn{2}{l}{} \\
\hline *Assessment of disease activity using Crohn's disease activity index \\
(CDAI) in CD and clinical activity index (CAl) in UC. \\
5-ASA, 5-aminosalicylic acid, SASP, salicylazosulphapyridine.
\end{tabular}

incubated in medium containing $0.75 \mathrm{mM}$ EDTA (Sigma) for 60 minutes at $37^{\circ} \mathrm{C}$. During this treatment, intraepithelial lymphocytes and epithelial cells were removed from the tissue. Then, tissues that contained LPMCs were collected and incubated in medium containing $0.02 \%$ collagenase (Worthington Biochemical Corp, Freehold, New Jersey, USA). The fraction was pelleted and cells centrifuged over a 40-60\% Percoll solution (Amersham Biosciences Corp, Piscataway, New Jersey, USA) density gradient. PBMCs were isolated by density gradient centrifugation using Lymphoprep (Nycomed Pharma, Oslo, Norway) from heparinised peripheral blood samples. LPMCs or PBMCs were further separated into CD4 positive cells using MACS (Miltenyi Biotec, Auburn, California, USA) according to the manufacturer's instructions.

\section{Cell culture}

LPMCs and CD4+ PBMCs were cultured at a concentration of $1 \times 10^{6} / \mathrm{ml}$ in complete RPMI 1640 medium (Sigma) supplemented with $10 \%$ fetal bovine serum, $100 \mathrm{U} / \mathrm{ml}$ penicillin, and $100 \mathrm{mg} / \mathrm{ml}$ streptomycin (Invitrogen). For CD4+ PBMCs culture, $10 \mu \mathrm{g} / \mathrm{ml}$ of immobilised anti-CD3 (OKT3) and $5 \mu \mathrm{g} /$ $\mathrm{ml}$ of anti-CD28 (CD28.2; BD Pharmingen, San Diego, California, USA) antibodies were used. Recombinant IL-12p70 (BD Pharmingen) and/or IL-18 (MBL, Nagoya, Japan) were added in culture medium, as indicated.

\section{Enzyme linked immunosorbent assay (ELISA)}

Concentrations of IFN- $\gamma$ and IL- 12 in culture supernatants of LPMCs and PBMCs were measured using specific ELISA (IFN- $\gamma$ : Endogen, Woburn, Massachusetts, USA, IL-12: BioSource, Camarillo, California, USA). According to the manufacturer's instructions, the minimum detectable IL-12 and IFN- $\gamma$ concentrations were $7.8 \mathrm{pg} / \mathrm{ml}$ and $25.6 \mathrm{pg} / \mathrm{ml}$, respectively.

\section{Flow cytometry}

Flow cytometric analysis was performed as previously described. ${ }^{9}$ Phycoerythrin conjugated antihuman IL- 12 receptor $\beta 2$ (IL-12R $\beta 2$ ) antibody was purchased from BD Pharmingen. Fluorescence intensity on the surface of the cells was analysed using a FACScan (Becton Dickinson, Mountain View, California, USA).

\section{Protein extraction and western blotting}

Total protein was extracted using lysis buffer containing $10 \mathrm{mM}$ Tris $\mathrm{HCl}, 150 \mathrm{mM} \mathrm{NaCl}, 1 \mathrm{mM}$ EDTA, 0.5\% NP-40, and a mixture of protease inhibitors. Total protein $(10 \mu \mathrm{g})$ was separated on a NuPAGE 4-12\% Bis-Tris gel (Invitrogen) and electrophoretically transferred onto Immobilon-P membrane (Millipore, Bedford, Massachusetts, USA). To detect T-bet protein, the membrane was incubated with rabbit antiT-bet antisera ( 1:3000 final dilution; kindly provided by Drs L Glimcher and S Szabo, Harvard, School of Public Health, Boston, Massachusetts, USA) and subsequently with horseradish peroxidase conjugated goat antirabbit IgG Ab (1:2000, New England Biolabs, Beverly, Massachusetts, USA). Antibody reactions were detected with a chemiluminescence detection kit (Amersham Biosciences Corp). The membrane was subsequently stripped with Restore western blot stripping buffer (Pierce, Rockford, Illinois, USA) and incubated with mouse anti- $\beta$-actin Ab (1:2000, Sigma). Densitometric analysis was performed with NIH image software version 1.61 and T-bet expression was adjusted to $\beta$-actin expression.

\section{Statistical analysis}

Statistical differences were analysed using the MannWhitney $U$ test. A p value of $<0.05$ was considered to be significant. All data are expressed as mean (SEM). 


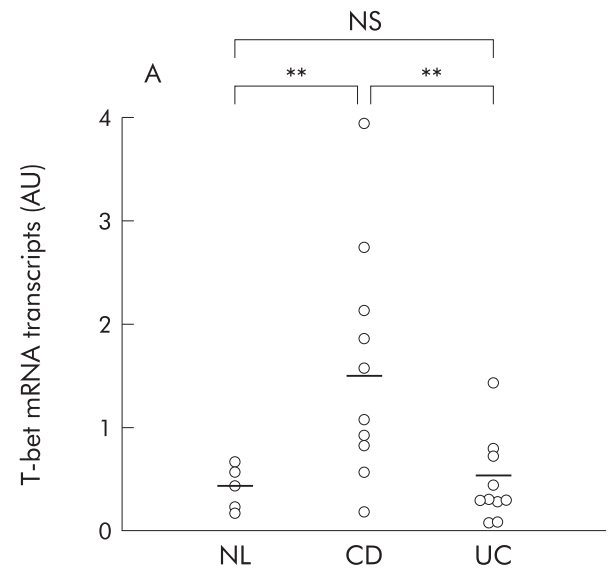

B
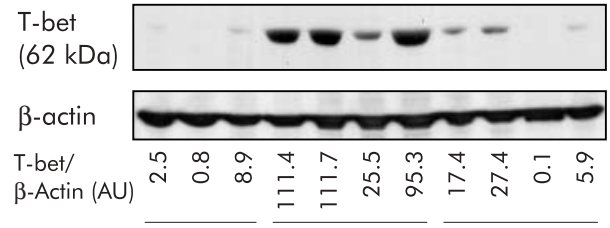

NL

UC

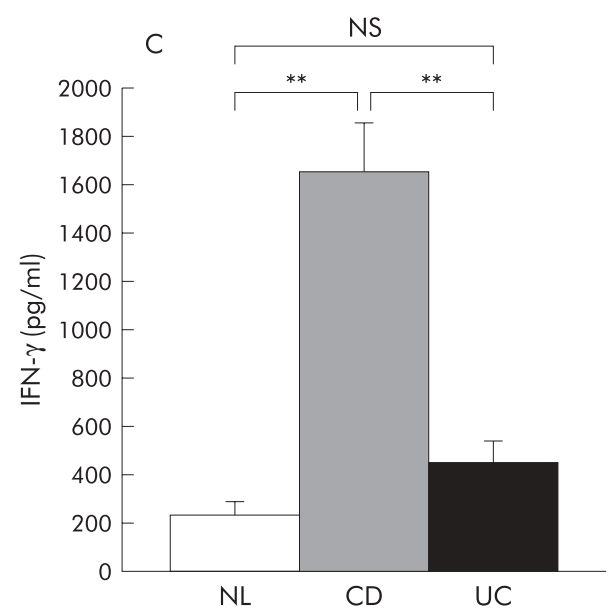

Figure 1 T-box expressed in T cells (T-bet) expression of CD4+ lamina propria mononuclear cells (LPMCS) was increased in Crohn's disease (CD). Total RNA was extracted from CD4+ LPMCs (CD, $n=10$; ulcerative colitis (UC), $n=10$; normal controls (NL), $n=5$ ). (A) Levels of T-bet mRNA transcripts were measured by quantitative real time polymerase chain reaction and adjusted to $\beta$-actin mRNA transcripts. ${ }^{* *} p<0.01$. (B) T-bet protein expression was assessed by western blotting in CD and UC patients and in NL. AU, arbitrary units. (C) LPMCs were cultured in medium alone for 48 hours ( $C D, n=6 ; U C, n=6 ; N L, n=6$ ). Production of interferon $\gamma$ (IFN- $\gamma$ ) was measured by ELISA. ${ }^{* *} \mathrm{p}<0.01$.

\section{RESULTS}

\section{T-bet was upregulated in CD4+ LPMCs of CD}

To clarify the involvement of T-bet in Thl mediated immunopathology in CD, we first assessed expression of T-bet mRNA in CD4+ LPMCs obtained from five NL, 10 CD patients, and $10 \mathrm{UC}$ patients using quantitative real time PCR. T-bet mRNA obtained from CD4+ LPMCs of CD patients

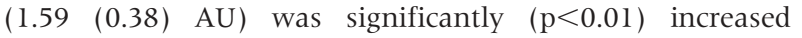
compared with that obtained from UC patients $(0.48(0.14)$ $\mathrm{AU})$ and NL (0.41 (0.11) AU) (fig 1A). T-bet protein was also significantly $(\mathrm{p}<0.05)$ increased in CD4+ LPMCs of CD patients (88.5 (21.3) AU) compared with those of UC patients
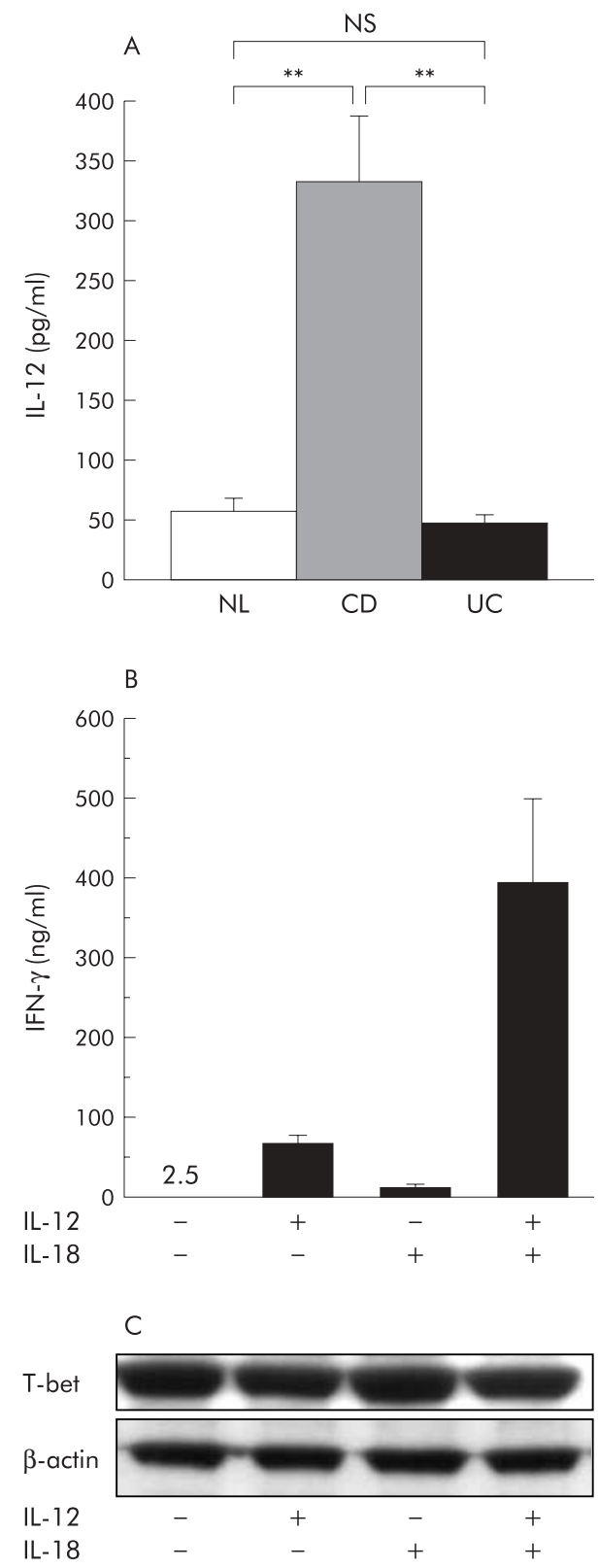

Figure 2 T-box expressed in T cells (T-bet) did not contribute to synergistic augmentation of interferon $\gamma($ IFN- $\gamma$ ) production by interleukin (IL)-12 and IL-18 stimulation. (A) Lamina propria mononuclear cell (LPMCs) were cultured in medium alone for 48 hours. Production of IL-12 was measured by ELISA in Crohn's disease (CD) and ulcerative colitis (UC) patients, and in normal controls (NL). ${ }^{* *} p<0.01$. (B) LPMCs from CD patients were stimulated by IL-12 $(1 \mathrm{ng} / \mathrm{ml})$ and/or IL-18 $(1 \mathrm{ng} / \mathrm{ml}$ ) for 48 hours. Production of IFN- $\gamma$ was measured by ELISA $(n=6)$. (C) T-bet protein expression was assessed by western blotting. Results are representative of three independent experiments.

(12.7 (7.2) AU) and NL (4.1 (3.1) AU) (fig 1B). T-bet expression was not significantly different between UC and NL at both the mRNA and protein levels.

To assess the correlation between T-bet expression and Thl responses, we next examined IFN- $\gamma$ production by LPMCs cultured without any stimuli for 48 hours, as measured by ELISA. As shown in fig $1 C$, IFN- $\gamma$ production by LPMCs from patients with $C D$ was significantly $(p<0.01)$ higher $(1659.0$ (206.7) $\mathrm{pg} / \mathrm{ml}$ ) than that from patients with UC (461.5 (88.1) $\mathrm{pg} / \mathrm{ml}$ ) or from NL (233.3 (61.4) pg/ml). This enhanced production of IFN- $\gamma$ well corresponded to augmentation of 
A

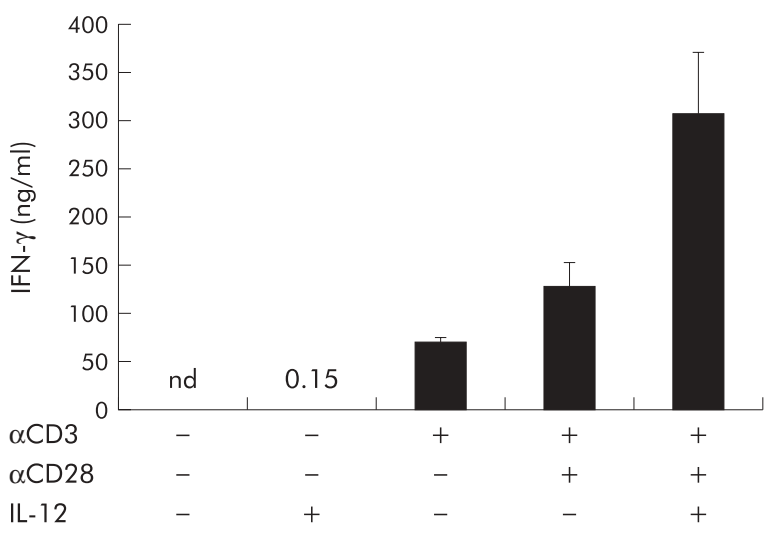

C

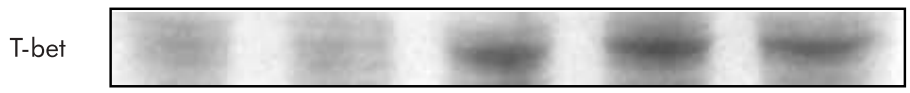

$\beta$-actin

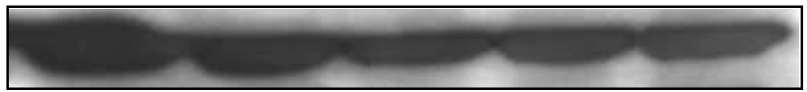

$\alpha \mathrm{CD} 3$

$\alpha C D 28$

IL-12
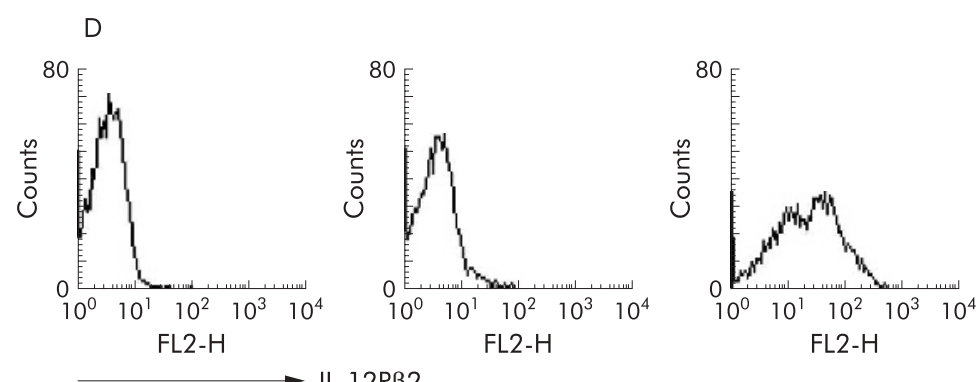

IL-12Rß2

$+\quad+$

$+\quad+$

- $\quad+$ $\alpha \mathrm{CD} 3$

$\alpha \mathrm{CD} 28$

IL-12
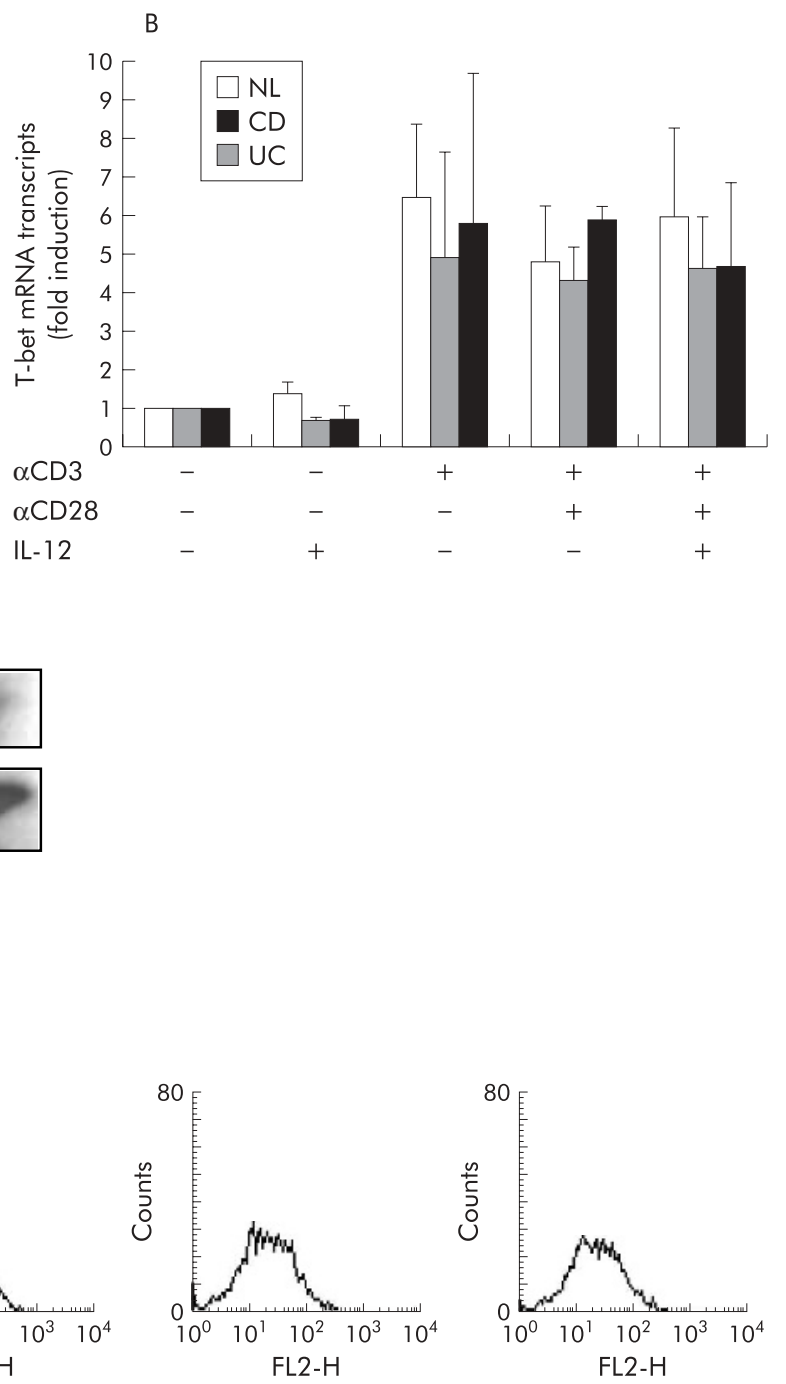

$+$

$+$

$+$

$+$

Figure 3 Induction of T-box expressed in T cells (T-bet) via the T cell receptor (TCR) signalling pathway is necessary for interferon $\gamma$ (IFN- $\gamma$ ) production before interleukin (IL)-12 stimulation. CD4+ PBMCs were stimulated with anti-CD3 (10 $\mu \mathrm{g} / \mathrm{ml}) / C D 28(5 \mu \mathrm{g} / \mathrm{ml})$ and/or IL-12 (10 ng/ml) for 48 hours. (A) Production of IFN- $\gamma$ was measured by ELISA $(n=3)$. (B) T-bet mRNA expression was analysed by quantitative real time polymerase chain reaction after stimulation for 12 hours in patients with Crohn's disease (CD) and ulcerative colitis (UC), and in normal controls (NL). (C) T-bet protein expression was assessed by western blotting. (D) Surface expression of IL-12Rß2 was analysed by FACS.

T-bet expression by LPMCs from patients with CD. These results indicated that $\mathrm{T}$-bet may essentially contribute to Thl immune responses in CD.

\section{T-bet did not contribute to synergistic augmentation of IFN- $\gamma$ production by IL- 12 and IL- 18 stimulation}

To investigate which stimuli induce T-bet in CD, we focused on Thl inducing cytokines such as IL-12, which is secreted by activated macrophages and dendritic cells via activation of innate immune responses. We first measured spontaneous IL- 12 production from LPMCs. As previously reported, ${ }^{24}$ IL- 12 production by LPMCs from patients with CD (333.3 (54.7) $\mathrm{pg} / \mathrm{ml}$ ) was significantly $(\mathrm{p}<0.01)$ higher than that from patients with UC $(48.4(7.1) \mathrm{pg} / \mathrm{ml})$ or NL $(56.7$ (11.4) $\mathrm{pg} / \mathrm{ml}$ ) (fig 2A). We next examined whether IL-12 and/or another Thl inducing cytokine IL-18 could regulate T-bet expression in LPMCs from patients with CD. While
IL-12 or IL-18 by themselves increased IFN- $\gamma$ production by LPMCs from patients with CD, in combination a dramatic effect on IFN- $\gamma$ production by cells was noted. However, neither single nor combined addition of IL-12/IL-18 significantly upregulated T-bet expression (fig 2C). These results demonstrate that synergistic augmentation of IFN- $\gamma$ production by IL- 12 and IL-18 in CD patients did not require further T-bet upregulation, suggesting a T-bet independent pathway.

Induction of T-bet via the T cell receptor (TCR) signalling pathway is necessary for IFN- $\gamma$ production before IL-12 stimulation

Next, we hypothesised that TCR stimulation was important for T-bet induction early in Thl mediated immunopathology in CD. Because LPMCs are reported to show activated phenotypes, ${ }^{2}$ indicating that they have already experienced antigenic stimulation in the intestinal mucosa, we used CD4+ 
PBMCs in the following experiments. CD4+ PBMCs were stimulated with plate bound anti-CD3 and/or CD28 mAb in the presence or absence of IL-12. After in vitro activation, IFN $-\gamma$ production and T-bet expression were analysed. As shown in fig 3A, CD4+ PBMCs from CD patients produced little IFN- $\gamma$ in the presence of IL-12 without plate bound antiCD3 mAb stimulation. Under these conditions, T-bet was not augmented at either the mRNA or protein level (fig 3B, C). In contrast, anti-CD3 mAb stimulation without exogenous IL-12 induced a large amount of IFN- $\gamma(68.5(5.0) \mathrm{ng} / \mathrm{ml})$, as well as marked expression of T-bet (fig 3A-C). The level of T-bet induction by anti-CD3 mAb stimulation was not significantly different among CD4+ PBMCs from CD or UC patients, or NL (fig 3B). Consistent with mRNA expression, T-bet protein was augmented by anti-CD3 mAb stimulation in CD4+ PBMCs from patients with CD (fig 3C; similarly in UC and NL, but data not shown). Furthermore, in parallel with T-bet induction by anti-CD3 mAb stimulation, IL-12R $\beta 2$ was also induced in CD4+ PBMCs (fig 3D) and additional stimulation by IL- 12 induced a fivefold greater amount of IFN- $\gamma(355.0$ (62.2) $\mathrm{ng} / \mathrm{ml}$ ) than anti-CD3 mAb stimulation alone (fig 3A). However, IL-12 costimulation did not induce further T-bet upregulation (fig 3B, C) which was consistent with data regarding LPMCs shown in fig $2 \mathrm{C}$. It is reported that transcription of IL-12R 32 was regulated by T-bet in mice. ${ }^{25} 26$ Collectively, these results indicate that T-bet is induced through TCR stimulation prior to IL-12 signalling for enhanced IFN- $\gamma$ production; T-bet may not only initiate IFN- $\gamma$ production but also control the responsiveness to IL- 12 through upregulation of IL-12R $\beta 2$.

\section{DISCUSSION}

Although CD represents typical Thl mediated immune responses, the molecular mechanism involved in differentiation of Thl cells in CD has not been elucidated. T-bet has been proposed to be the master regulator of Thl development based on its induction of IFN- $\gamma$ and repression of Th2 cytokines. ${ }^{19}$ Furthermore, a study in mice showed that T-bet was STAT-4 independent and acted before IL-12 in Thl development. ${ }^{25}$ These observations led us to consider the role of T-bet in human inflammatory bowel diseases, especially in CD. Here we demonstrated that T-bet was expressed strongly in CD4+ LPMCs from CD patients compared with those from UC patients and NL. Neurath et al demonstrated that T-bet was strongly expressed in CD3+ LPMCs in patients with CD while only weak expression was observed in NL and patients with UC. ${ }^{21}$ A definitive conclusion could not be drawn from their results because CD3+ T lymphocytes included both $\mathrm{CD} 4+$ and CD8+ T lymphocytes, and T-bet is critical for IFN- $\gamma$ production in CD4+ but not in CD8+ T lymphocytes. ${ }^{20}$ Accordingly, we focused on isolated CD4+ LPMCs to study the role of T-bet in induction of Thl immune responses in CD. In this study, we showed upregulation of T-bet in CD4+ LPMCs from CD. Increased expression of $\mathrm{T}$-bet in $\mathrm{CD}$ was consistent with enhanced production of IFN- $\gamma$ from LPMCs of CD. Collectively, these results indicate that T-bet may play an essential role in induction of Thl immune responses in CD.

What regulates T-bet augmentation in CD? We demonstrated that induction of T-bet in human CD4+ PBMCs was mediated by anti-CD3 mAb, but not IL-12, suggesting that antigenic stimulation was essential for T-bet induction. Although some studies have suggested that IFN- $\gamma /$ STAT- 1 signalling is the key pathway for T-bet expression, ${ }^{26} 27$ another study revealed that TCR signalling could maintain T-bet expression in committed CD4+ cells. $^{28}$ The result indicated that repeated antigenic stimulations were essential for retaining high T-bet expression. It is well known that the gastrointestinal tract is continuously exposed to a large amount of antigens derived from the diet, bacteria, and pathogens. Sustained dysregulated $\mathrm{T}$ cell responses to ubiquitous antigens could result in continuous upregulation of T-bet in CD. Hyporesponsiveness (tolerance) against various luminal antigens in normal intestinal mucosa is maintained through anti-inflammatory cytokines such as transforming growth factor- $\beta$ (TGF- $\beta$ ). It was reported that TGF- $\beta$ suppressed T-bet expression. ${ }^{21}{ }^{29}$ Considering that mucosal T cells from CD patients have been shown to be insensitive to TGF- $\beta$ inhibition, ${ }^{30}$ disruption of TGF- $\beta$ signalling may possibly be involved in overexpression of T-bet as well as hyperreactivity against various luminal antigens. These findings are consistent with the finding that elimination of luminal antigens by elemental diet or decontamination of gut flora can attenuate mucosal inflammation in human CD and murine experimental colitis models. ${ }^{31-34}$

Although induction of T-bet by anti-CD3 mAb in CD4+ PBMCs was not different between $\mathrm{CD}$ and UC, increased expression of T-bet was observed only in CD4+ LPMCs from patients with $C D$. These data suggest that the cytokine environment in UC is different from that in CD (that is, increased production of IL-5, which is a typical Th2 cytokine). ${ }^{5}$ It was reported that Th2 skewing conditions suppressed T-bet expression. ${ }^{19}$ Therefore, in vivo T-bet expression in the presence of chronic gut inflammation may be influenced by the local environment, including cytokine patterns. Further studies would however be necessary to clarify these points.

What then is the role of T-bet in induction of Thl mediated immunopathology in CD? In the present study, we demonstrated that IL-12 in itself could not induce IFN- $\gamma$ production from CD4+ PBMCs that did not express T-bet. In contrast, anti-CD3 mAb stimulation induced T-bet expression in CD4+ PBMCs and caused them to produce IFN- $\gamma$. Activated CD4+ PBMCs also increased IL-12R $\beta 2$ and responded to IL-12 for further production of IFN- $\gamma$. It has been reported that transcription of IL-12R $\beta 2$ is regulated by T-bet in mice. ${ }^{25} 26$ We and other investigators have previously demonstrated that LPMCs from CD patients expressed high levels of IL-12R $\beta 2$ and responded to IL-12 more strongly than those from NL. ${ }^{35}{ }^{36}$ Consistent with previous reports, our data indicate that T-bet is necessary for initiating IFN- $\gamma$ production, and that it regulates IL-12 responsiveness through IL-12R $\beta 2$ upregulation in CD.

We have demonstrated here that T-bet expression was increased in CD4+ LPMCs from patients with CD. T-bet induced by antigens in the gut lumen initiates IFN- $\gamma$ production and modulates IL-12 responsiveness through upregulation of IL-12R $\beta 2$. Furthermore, dysregulated production of IL-12/IL-18, as observed specifically in CD patients in our previous and present studies, may intensify Thl polarisation of the majority of $\mathrm{T}$ cells initiated by T-bet. Thus serial actions by antigenic stimulation to induce T-bet in LPMCs and macrophage derived IL-12/18 could be essential for establishing Thl mediated immunopathology in CD.

\section{ACKNOWLEDGEMENTS}

We thank Professor Daniel Podolsky and Dr Cosmas Giallourakis for critical comments, Drs L Glimcher and S Szabo for providing the antibodies, Professor M Kitajima and Drs M Watanabe, H Hasegawa, $\mathrm{Y}$ Iwao, and M Naganuma for providing the specimens, and $\mathrm{Dr} \mathrm{H}$ Takaishi for helpful discussion. This work was supported in part by grants-in-aid from the Japanese Ministry of Education, Culture, and Science, the Japanese Ministry of Labor, Health, and Welfare, Japan Health Sciences Foundation, Keio University, and Keio University Medical Fund.

\footnotetext{
Authors' affiliations

K Matsuoka, N Inove, T Sato, S Okamoto, T Hisamatsu, Y Kishi,

A Sakuraba, O Hitotsumatsu, H Ogata, H Ishii, T Hibi, Department of Internal Medicine, School of Medicine, Keio University, Tokyo, Japan
} 
K Koganei, T Fukushima, Department of Surgery, Yokohama Municipal Citizen's Hospital, Yokohama, Japan

T Kanai, M Watanabe, Department of Gastroenterology and Hepatology, Graduate School, Tokyo Medical and Dental University, Tokyo, Japan

\section{REFERENCES}

Neurath MF, Finotto S, Glimcher LH. The role of Th1/Th2 polarization in mucosal immunity. Nat Med 2002;8:567-73.

2 Fiocchi C. Inflammatory bowel disease: etiology and pathogenesis. Gastroenterology 1998;115:182-205.

3 Kanai T, Watanabe $M$, Okazawa A, et al. Interleukin 18 is a potent proliferative factor for intestinal mucosal lymphocytes in Crohn's disease. Gastroenterology 2000;119:1514-23.

4 Monteleone I, Vavassori P, Biancone L, et al. Immunoregulation in the gut: success and failures in human disease. Gut 2002:50:1160-4.

5 Fuss IJ, Neurath M, Boirivant M, et al. Disparate CD4+ lamina propria (LP) lymphokine secretion profiles in inflammatory bowel disease. Crohn's disease LP cells manifest increased secretion of IFN-gamma, whereas ulcerative colitis LP cells manifest increased secretion of IL-5. J Immunol 1996;157:1261-70.

6 Pizarro $\Pi$, Michie MH, Bentz M, et al. IL-18, a novel immunoregulatory cytokine, is up-regulated in Crohn's disease: expression and localization in intestinal mucosal cells. J Immunol 1999;162:6829-35.

7 Monteleone G, Trapasso F, Parrello T, et al. Bioactive IL-18 expression is upregulated in Crohn's disease. J Immunol 1999:163:143-7.

8 Powrie F, Leach MW, Mauze S, et al. Inhibition of Th1 responses prevents inflammatory bowel disease in scid mice reconstituted with CD45RBhi CD4+ T cells. Immunity 1994;1:553-62

9 Kanai T, Watanabe M, Okazawa A, et al. Macrophage-derived IL-18mediated intestinal inflammation in the murine model of Crohn's disease. Gastroenterology 2001;121:875-88.

10 Neurath MF, Fuss I, Kelsall BL, et al. Antibodies to interleukin 12 abrogate established experimental colitis in mice. J Exp Med 1995;182:1281-90.

11 Targan SR, Hanquer SB, van Deventer SJ, et al. A short-term study of chimeric monoclonal antibody CA2 to tumor necrosis factor alpha for Crohn's disease. Crohn's Disease cA2 Study Group. N Engl J Med 1997;337:1029-35.

12 Present DH, Rutgeerts P, Targan S, et al. Infliximab for the treatment of fistulas in patients with Crohn's disease. N Engl J Med 1999;340:1398-405.

13 Glimcher LH, Murphy KM. Lineage commitment in the immune system: the T helper lymphocyłe grows up. Genes Dev 2000;14:1693-711

14 Rengarajan J, Szabo SJ, Glimcher LH. Transcriptional regulation of Th1/Th2 polarization. Immunol Today 2000;21:479-83.

15 Ho IC, Hodge MR, Rooney JW, et al. The proto-oncogene c-maf is responsible for tissue-specific expression of interleukin-4. Cell 1996;85:973-83.

16 Zheng W, Flavell RA. The transcription factor GATA-3 is necessary and sufficient for Th2 cytokine gene expression in CD4 T cells. Cell 1997;89:587-96.

17 Kurata H, Lee HJ, O'Garra A, et al. Ectopic expression of activated Stat6 induces the expression of Th2-specific cytokines and transcription factors in developing Th1 cells. Immunity 1999;11:677-88.
18 Farrar JD, Murphy KM. Type I interferons and T helper development. Immunol Today 2000;21:484-9.

19 Szabo SJ, Kim ST, Costa GL, et al. A novel transcription factor, T-bet, directs Th1 lineage commitment. Cell 2000;100:655-69.

20 Szabo SJ, Sullivan BM, Stemmann C, et al. Distinct effects of T-bet in TH1 lineage commitment and IFN-gamma production in CD4 and CD8 T cells. Science 2002;295:338-42.

21 Neurath MF, Weigmann B, Finotto S, et al. The transcription factor T-bet regulates mucosal T cell activation in experimental colitis and Crohn's disease. $J$ Exp Med 2002;195:1129-43.

22 Gower-Rousseau C, Salomez JL, Dupas JL, et al. Incidence of inflammatory bowel disease in northern France (1988-1990). Gut 1994;35:1433-8.

23 Lennard-Jones JE. Classification of inflammatory bowel disease. Scand J Gastroenterol Suppl 1989;170:2-6.

24 Monteleone G, Biancone L, Marasco R, et al. Interleukin 12 is expressed and actively released by Crohn's disease intestinal lamina propria mononuclear cells. Gastroenterology 1997:112:1169-78.

25 Mullen AC, High FA, Hutchins AS, et al. Role of T-bet in commitment of TH1 cells before IL-12-dependent selection. Science 2001;292:1907-10.

26 Afkarian M, Sedy JR, Yang J, et al. T-bet is a STATI-induced regulator of IL12R expression in naive CD4+ T cells. Nat Immunol 2002;3:549-57.

27 Lighvani AA, Frucht DM, Jankovic D, et al. T-bet is rapidly induced by interferon-gamma in lymphoid and myeloid cells. Proc Natl Acad Sci U S A 2001;98:15137-42.

28 Bajenoff $M$, Wurtz O, Guerder S. Repeated antigen exposure is necessary for the differentiation, but not the initial proliferation, of naive CD4(+) T cells. $J$ Immunol 2002;168:1723-9.

29 Gorelik L, Constant S, Flavell RA. Mechanism of transforming growth factor beta-induced inhibition of Thelper type 1 differentiation. J Exp Med 2002; 195: 1499-505.

30 Monteleone G, Kumberova A, Croft NM, et al. Blocking Smad7 restores TGFbetal signaling in chronic inflammatory bowel disease. J Clin Invest 2001;108:601-9.

31 Aranda R, Sydora BC, McAllister PL, et al. Analysis of intestinal lymphocytes in mouse colitis mediated by transfer of CD4+, CD45RBhigh T cells to SCID recipients. J Immunol 1997; 158:3464-73.

32 Kuhn R, Lohler J, Rennick D, et al. Interleukin-10-deficient mice develop chronic enterocolitis. Cell 1993;75:263-74.

33 Lochs H, Steinhardt HJ, Klaus-Wentz B, et al. Comparison of enteral nutrition and drug treatment in active Crohn's disease. Results of the European Cooperative Crohn's Disease Study. IV. Gastroenterology 1991;101:881-8.

34 Steinhart AH, Feagan BG, Wong CJ, et al. Combined budesonide and antibiotic therapy for active Crohn's disease: a randomized controlled trial. Gastroenterology 2002;123:33-40

35 Okazawa A, Kanai T, Watanabe M, et al. Th1-mediated intestinal inflammation in Crohn's disease may be induced by activation of lamina propria lymphocytes through synergistic stimulation of interleukin-12 and interleukin-18 without T cell receptor engagement. Am J Gastroenterol 2002;97:3108-17.

36 Parrello T, Monteleone G, Cucchiara S, et al. Up-regulation of the IL-12 receptor beta 2 chain in Crohn's disease. J Immunol 2000;165:7234-9.

\section{EDITOR'S QUIZ: GI SNAPSHOT}

\section{Answer}

From question on page 1302

The peripheral blood smear revealed numerous deformed red blood cells with thorny projections, consistent with spur cells. The increased free cholesterol/phospholipid molar ratio in the erythrocyte membrane was also noted. Magnetic resonance imaging demonstrated diffuse hypointensity of the liver, suggesting heavy iron loading. Spur cell anaemia of alcoholic cirrhosis was diagnosed.

Spur cell anaemia (acanthocytosis), a rare acquired haemolytic anaemia observed mainly in the end stages of alcoholic cirrhosis, is characterised by an increased ratio of free cholesterol to phospholipid in the erythrocyte membranes that results in multispiculated erythrocytes (acanthocytes). These acanthocytes undergo rapid splenic destruction and consequently have a shortened survival. Recent studies have indicated that alcoholic iron overload may be associated with spur cell anaemia rather than hereditary haemochromatosis. Patients usually need frequent blood transfusions and the prognosis is extremely poor. Liver transplantation, which improves hepatic function and resolves spur cell anaemia, has been the most effective treatment. Our patient did not choose liver transplantation. She had a poor response to conservative treatment with multiple blood transfusions and died of liver failure seven months later.

doi: $10.1136 /$ gut.2003.034785 